Supplement of Biogeosciences, 12, 4483-4496, 2015

http://www.biogeosciences.net/12/4483/2015/

doi:10.5194/bg-12-4483-2015-supplement

(C) Author(s) 2015. CC Attribution 3.0 License.

(c) (i)

Supplement of

\title{
Microbial assemblages on a cold-water coral mound at the SE Rockall Bank (NE Atlantic): interactions with hydrography and topography
}

\section{J. D. L. van Bleijswijk et al.}

Correspondence to: F. Mienis (furu.mienis@nioz.nl)

The copyright of individual parts of the supplement might differ from the CC-BY 3.0 licence. 


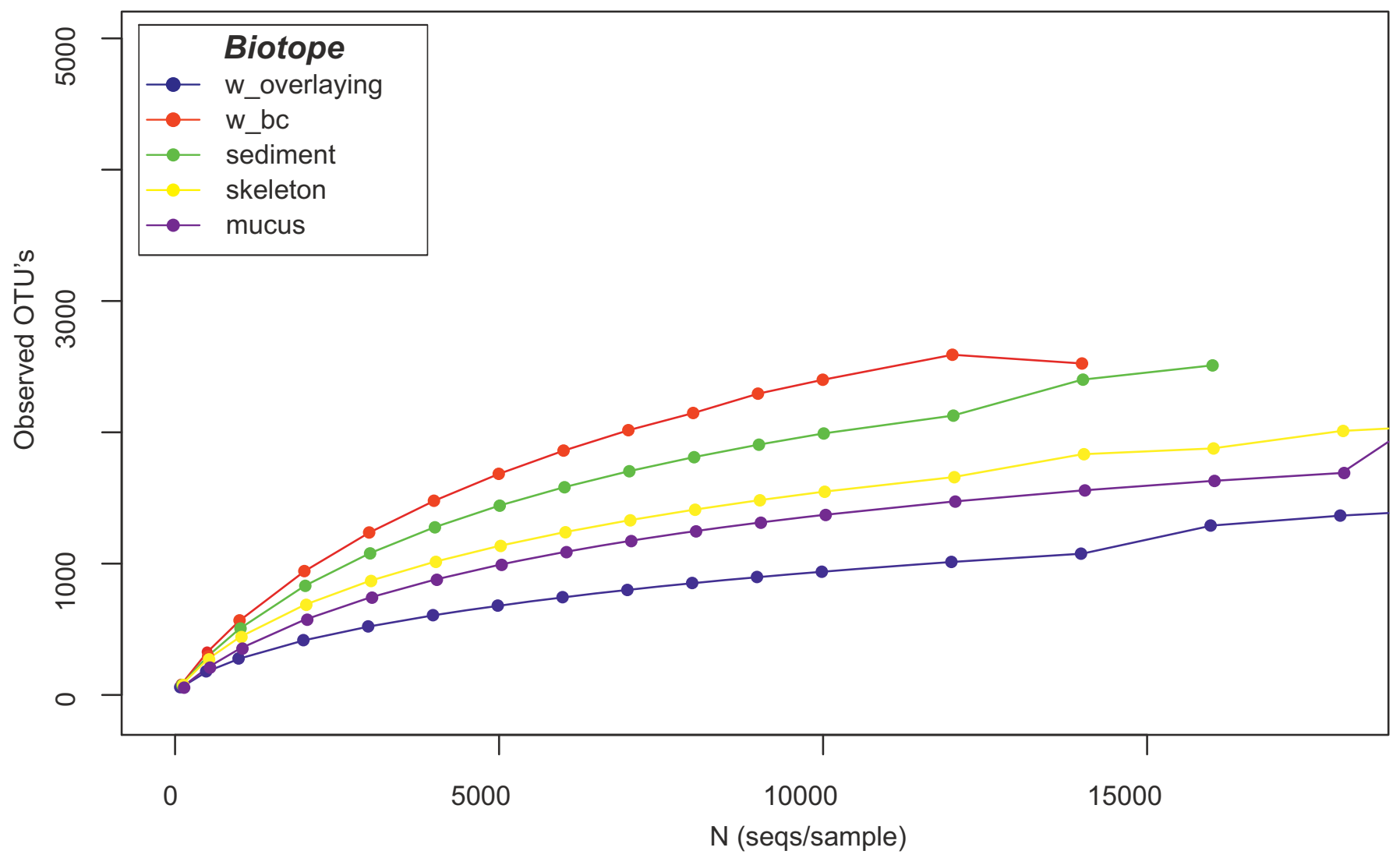

S1 


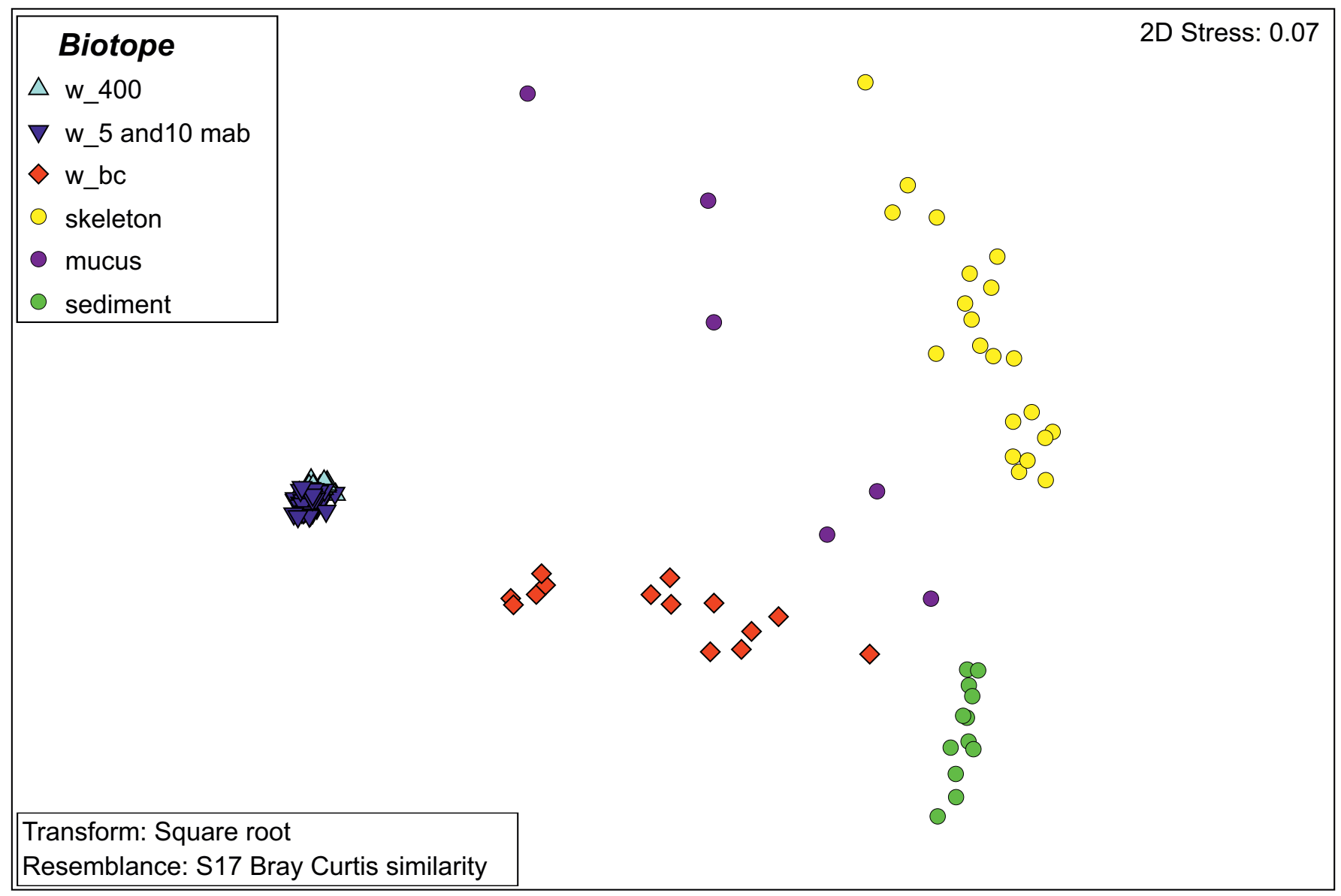

S2 
S1. Sequence output and microbial diversity indices (average \pm standard error) of five biotopes sampled at Haas Mound. Singletons were not excluded in this analysis.

\begin{tabular}{|l|l|l|l|l|}
\hline biotope & reads/sample & observed OTUs & Chao1 & Shannon \\
\hline $\begin{array}{l}\text { overlaying water } \\
(n=23)\end{array}$ & $17651 \pm 1599$ & $1712 \pm 119$ & $2684 \pm 306$ & $5.04 \pm 0.06$ \\
\hline $\begin{array}{l}\text { near-bottom water } \\
(n=5)\end{array}$ & $11456 \pm 798$ & $3858 \pm 567$ & $7876 \pm 618$ & $6.95 \pm 0.09$ \\
\hline $\begin{array}{l}\text { sediment } \\
(n=4)\end{array}$ & $14070 \pm 941$ & $3245 \pm 104$ & $5357 \pm 688$ & $6.40 \pm 0.16$ \\
\hline $\begin{array}{l}\text { skeleton } \\
(n=6)\end{array}$ & $17713 \pm 1952$ & $2856 \pm 300$ & $4637 \pm 709$ & $6.19 \pm 0.07$ \\
\hline $\begin{array}{l}\text { mucus } \\
(n=2)\end{array}$ & $20140 \pm 2229$ & $2663 \pm 665$ & $2828 \pm 1123$ & $4.93 \pm 0.87$ \\
\hline
\end{tabular}


S2. Indicator taxa given for five biotopes sampled at Haas Mound. Only those with the highest statistics values are listed. Numbers between brackets are number of strong indicators $(A>0.85)$ over the total number of significant indicators $(p<0.0001)$ found. $w_{-}$CTD $=$water sampled at $400 \mathrm{~m}$ and 5 and 10 mab; Near-bottom water ( $w_{-}$bc). $A$ = given the indicator is present, the probability that the sample belongs to the sample group. $\mathrm{B}=$ taking one sample from the group, the probability that it contains the indicator.

\begin{tabular}{|c|c|c|c|c|c|c|}
\hline $\begin{array}{l}\text { Sample group } \\
\text { (\#strong } \\
\text { indicators) }\end{array}$ & Indicator & A & B & stat & p.value & $\begin{array}{c}\text { Reads } \\
\text { avg \% in } \\
\text { sample } \\
\text { group }\end{array}$ \\
\hline \multirow[t]{5}{*}{ w_CTD $(4 / 38)$} & uncl. SAR11 clade Deep 1 & 0.8833 & 1.0000 & 0.940 & 0.0001 & 2.61 \\
\hline & $\begin{array}{c}\text { Rhodospirillaceae } \\
\text { AEGEAN-169 marine group }\end{array}$ & 0.8796 & 1.0000 & 0.938 & 0.0001 & 2.20 \\
\hline & $\begin{array}{c}\text { uncl. Verrucomicrobia } \\
\text { Arctic97B-4 marine group }\end{array}$ & 0.8751 & 1.0000 & 0.935 & 0.0001 & 0.45 \\
\hline & $\begin{array}{c}\text { uncl. Thermoplasmatales } \\
\text { Marine Group III }\end{array}$ & 0.8721 & 1.0000 & 0.934 & 0.0001 & 1.00 \\
\hline & $\begin{array}{l}\text { uncl. Oceanospirillales } \\
\text { ZD0405 }\end{array}$ & 0.8361 & 1.0000 & 0.914 & 0.0001 & 2.85 \\
\hline \multirow[t]{8}{*}{ w_bc (8/13) } & $\begin{array}{l}\text { uncl. Dehalococcoidia } \\
\text { vadinBA26 }\end{array}$ & 0.9437 & 1.0000 & 0.971 & 0.0001 & 0.36 \\
\hline & $\begin{array}{c}\text { uncultured } \\
\text { Oceanospirillaceae }\end{array}$ & 0.9460 & 0.8571 & 0.900 & 0.0001 & 0.05 \\
\hline & uncl. Dehalococcoidia GIF3 & 1.0000 & 0.7143 & 0.845 & 0.0001 & 0.27 \\
\hline & uncl. BHI80-139 & 0.8931 & 0.7857 & 0.838 & 0.0001 & 0.07 \\
\hline & $\begin{array}{l}\text { uncl. Dehalococcoidia } \\
\text { Sh765B-AG-111 }\end{array}$ & 1.0000 & 0.6429 & 0.802 & 0.0001 & 0.09 \\
\hline & $\begin{array}{c}\text { Sphingobacteriales KD1- } \\
131\end{array}$ & 0.8881 & 0.7143 & 0.796 & 0.0001 & 0.09 \\
\hline & Thaumarchaeota Group C3 & 1.0000 & 0.5714 & 0.756 & 0.0001 & 0.03 \\
\hline & Brocadiaceae W4 & 0.9982 & 0.5000 & 0.706 & 0.0001 & 0.83 \\
\hline
\end{tabular}




\begin{tabular}{|c|c|c|c|c|c|c|}
\hline sediment $(0 / 3)$ & Phycisphaerae C86 & 0.6982 & 1.0000 & 0.836 & 0.0001 & 0.25 \\
\hline & $\begin{array}{l}\text { uncl. Chloroflexi JG30-KF- } \\
\text { CM66 }\end{array}$ & 0.5118 & 1.0000 & 0.715 & 0.0001 & 0.56 \\
\hline & $\begin{array}{l}\text { uncl. Rhodospirillales AT- } \\
\text { s3-44 }\end{array}$ & 0.3669 & 1.0000 & 0.606 & 0.0001 & 0.32 \\
\hline \multirow[t]{3}{*}{ skeleton (0/12) } & uncul. Caldilineaceae & 0.7979 & 1.0000 & 0.893 & 0.0001 & 0.71 \\
\hline & Granulosicoccus & 0.7513 & 1.0000 & 0.867 & 0.0001 & 1.87 \\
\hline & Profundibacterium & 0.7602 & 0.9524 & 0.851 & 0.0001 & 0.22 \\
\hline \multirow[t]{12}{*}{ mucus $(12 / 12)$} & $\begin{array}{l}\text { uncl. Oceanospirillales } \\
\text { G02-CRO2-full }\end{array}$ & 0.9982 & 1.0000 & 0.999 & 0.0001 & 0.36 \\
\hline & Acinetobacter & 0.9872 & 1.0000 & 0.994 & 0.0001 & 9.11 \\
\hline & uncult. Helicobacteraceae & 0.9699 & 1.0000 & 0.985 & 0.0001 & 0.48 \\
\hline & $\begin{array}{l}\text { uncl. Oceanospirillales } \\
\text { BPS-CK174 }\end{array}$ & 0.9651 & 1.0000 & 0.982 & 0.0001 & 0.29 \\
\hline & $\begin{array}{l}\text { Alteromonadaceae BD1-7 } \\
\text { clade }\end{array}$ & 0.9636 & 1.0000 & 0.982 & 0.0001 & 22.00 \\
\hline & Corynebacterium & 0.9259 & 1.0000 & 0.962 & 0.0001 & 0.11 \\
\hline & Staphylococcus & 0.9169 & 1.0000 & 0.958 & 0.0001 & 0.06 \\
\hline & Sphingomonas & 0.9000 & 1.0000 & 0.949 & 0.0001 & 0.15 \\
\hline & Enhydrobacter & 0.9963 & 0.8333 & 0.911 & 0.0001 & 0.17 \\
\hline & Methylobacterium & 0.9705 & 0.8333 & 0.899 & 0.0001 & 0.24 \\
\hline & Tumebacillus & 0.9106 & 0.8333 & 0.871 & 0.0001 & 0.13 \\
\hline & Micrococcus & 0.9773 & 0.5000 & 0.699 & 0.0001 & 0.06 \\
\hline
\end{tabular}

